\title{
COUPLING LOSSES OF MULTIFILAMENTARY SUPERCONDUCTORS HAVING SEVERAL CONCENTRIC REGIONS AND MIXED MATRIX
}

\author{
G.B.J. MULDER ${ }^{*}$ AND E.M.J. NIESSEN ${ }^{\#}$
}

* Philips Medical Systems Nederland B.V., P.O. Box 10000, 5680 DA Best, The Netherlands.
Applied Superc. Centre, Univ. of Twente, P.O. Box 217, 7500 AE Enschede, The Netherlands.

Abstract -- A method is presented to calculate the coupling loss in multifilamentary conductors containing mixed matrix. The conductor geometry considered here has several concentric regions with either isotropic normal material, anisotropic normal material, or superconducting filaments. In anisotropic normal regions, containing for example $\mathrm{Cu}$ hexagons in a $\mathrm{CuNi}$ environment, the effects of anisotropy and twist have to be taken into account. An interesting similarity is observed between such regions with highly anisotropic normal material and regions with superconducting filaments.

\section{INTRODUCTION}

Technologically advanced low-loss multifilamentary conductors usually employ a mixed matrix, i.e. a combination of different normal materials. Here, the use of $\mathrm{Cu}$ and $\mathrm{CuNi}$ is assumed. The purpose of the mixed matrix is to obtain a poor electrical conductivity in the azimuthal direction (leading to a reduction of the coupling loss), and combine it with a good conductivity in axial direction (in order to improve the stabilization and protection). Often, the stabilizing $\mathrm{Cu}$ is present in the superconducting region, for example directly on the surface of the filaments. But the wire may also have stabilizing $\mathrm{Cu}$ in separate regions, in which $\mathrm{CuNi}$ barriers have been added to reduce the transverse conductivity, for example $\mathrm{Cu}$ hexagons in a $\mathrm{CuNi}$ environment. The latter conductor concept is of particular interest in this paper because then the presence of normal material with an anisotropic conductivity will have an important influence on the loss.

\section{BASIC EQUATIONS}

The general approach to calculate coupling losses in multifilamentary wires is to use Carr's continuum model [1] and solve Maxwell's equations $\nabla \times \mathbf{E}=-\partial_{t} \mathbf{B}$ and $\nabla \times\left(\mathbf{B}-\mu_{\mathrm{o}} \mathbf{M}\right)=\mu_{\mathrm{o}} \mathbf{j}$. After neglecting the term $\nabla \times \mathbf{M}$ we obtain in cylinder coordinates:

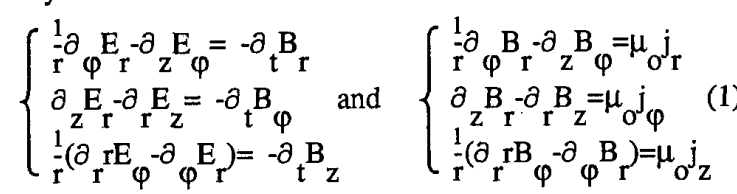

Solutions of (1) automatically satisfy $\nabla \cdot \mathbf{j}=0$ and $\nabla \cdot \mathbf{B}=0$.

Manuscript received August $24,1992$.
Due to the anisotropy of the composite, and its twist, the constitutive equation relating $\mathbf{j}$ to $\mathbf{E}$ becomes:

$$
\begin{aligned}
& \left\{\begin{array}{l}
j_{r}=\sigma_{\pi} E_{r}=\sigma_{\perp} E_{r} \\
j_{\varphi}=\sigma_{\varphi \varphi} E_{\varphi}+\sigma_{\varphi z} E_{z}+\eta_{s} j_{s} \sin \psi \\
j_{z}=\sigma_{z z} E_{z}+\sigma_{z \varphi} E_{\varphi}+\eta_{s} j_{s} \cos \psi
\end{array}\right. \\
& \text { with } \sigma_{\varphi \varphi}=\sigma_{\perp} \cos ^{2} \psi+\sigma_{\|} \sin ^{2} \psi \\
& \sigma_{\mathrm{zz}}=\sigma_{\|} \cos ^{2} \psi+\sigma_{\perp} \sin ^{2} \psi \\
& \sigma_{\varphi z}=\sigma_{z \varphi}=\left(\sigma_{\|}-\sigma_{\perp}\right) \sin \psi \cos \psi \text {, }
\end{aligned}
$$

where $\psi$ is the twist angle i.e. $\tan \psi=2 \pi \mathrm{r} / \mathrm{L}_{\mathrm{p}}$, and $\eta_{\mathrm{s}}$ the fraction of superconductor. The local conductivities transverse to and parallel with the filament direction are given by $\sigma_{\perp}$ and $\sigma_{\|}$. Note that the conductivity tensor $\overline{\bar{\sigma}}$ is a function of $r$. The superconducting nature of the filaments is expressed by a non-linear relation between $j_{s}$ and $E_{\|}$, with $j_{s}$ the sc. current density and $\mathrm{E}_{\|}$the electrical field along the filaments. The maximum value of $j_{s}$ is the critical current density $j_{c}$, which occurs when filaments are fully saturated. The presence of an electrical field $E_{\|}$does not necessarily imply that $j_{s}=j_{c}$, because under AC conditions the filaments have a dynamic resistivity proportional to their diameter and $\left|\dot{B}_{\perp}\right|$. We can approximate $j_{s}\left(E_{\|}\right)$as:

$$
\begin{aligned}
\mathrm{j}_{\mathrm{s}} & =\mathrm{j}_{\mathrm{c}} \operatorname{sign}\left(\mathrm{E}_{\|\|}\right) \text {if }\left|\mathrm{E}_{\|}\right| \geq \mathrm{E}^{*} \\
& =\mathrm{j}_{\mathrm{c}} \mathrm{E}_{\|} / \mathrm{E}^{*} \text { if }\left|\mathrm{E}_{\|}\right|^{*} \quad \text { with } \mathrm{E}^{*}=\frac{4}{3 \pi} \mathrm{d}_{\mathrm{fil}}\left|\dot{\mathrm{B}}_{\perp}\right| .
\end{aligned}
$$

If we can solve the system $(1,2,3)$, the coupling loss can be found by integrating $\mathbf{E} \cdot \mathbf{j}$ over time and volume. A few authors $[2,3]$ have done this numerically under the most general conditions, which is an important result for understanding the exact processes inside the wire. For most purposes, however, such a method is impractical and a simpler approach is desired.

To simplify the problem we make the following assumption. If $\mathbf{B}_{\mathbf{i}}$ is the induced magnetic field caused by coupling currents in the wire and $\mathbf{B}_{\mathrm{a}}$ is the externally applied field, then $\dot{\mathbf{B}}_{\mathrm{i}}$ is assumed to be negligible compared to $\dot{\mathbf{B}}_{\mathrm{a}}$. This condition holds at low frequencies when $\mathbf{B}_{\mathbf{a}}$ changes slowly compared to the decay time constant $\tau$ of the coupling currents. Actually, it corresponds to the quasi-stationary situation where $\mathbf{E}$ is directly proportional to $\left|\dot{\mathbf{B}}_{\mathrm{a}}\right|$. The applied field $\mathbf{B}_{\mathrm{a}}$ is taken transverse to the conductor, along $\varphi=0$. Using

$1051-8223 / 93 \$ 03.00 \odot 1993$ IEEE 
the $\varphi$-symmetry and $z$-invariance of the problem, we find that the most general form of $\mathbf{E}$ satisfying $(1,2)$ is

$$
\left\{\begin{array}{l}
\mathrm{E}_{r}(\mathrm{r}, \varphi, \mathrm{t})=\left(\dot{\mathrm{B}}_{\mathrm{a}} / \beta\right) \cdot \mathscr{E}_{\mathrm{r}}(\mathrm{r}) \cdot \cos \varphi \\
\mathrm{E}_{\varphi}(\mathrm{r}, \varphi, \mathrm{t})=\left(\dot{\mathrm{B}}_{\mathrm{a}} / \beta\right) \cdot \mathscr{E}_{\varphi}(\mathrm{r}) \cdot \sin \varphi \\
\mathrm{E}_{\mathrm{z}}(\mathrm{r}, \varphi, \mathrm{t})=\left(\dot{\mathrm{B}}_{\mathrm{a}} / \beta\right) \cdot \mathscr{E}_{\mathrm{z}}(\mathrm{r}) \cdot \sin \varphi
\end{array}\right.
$$

with $\beta=2 \pi / L_{p}$, and $\mathscr{E}$ a dimensionless function of $\mathrm{r}$ only. Substitution of (4) into (1) gives

$$
\begin{aligned}
& \mathscr{E}_{Z}=-\beta \mathrm{r} \\
& \partial_{\mathrm{r}} \mathrm{r} \mathscr{E}_{\varphi}+\mathscr{E}_{\mathrm{r}}=0 .
\end{aligned}
$$

The third equation, required to solve $(5,6)$, depends on the type of material, as will be explained below.

\section{LAY-OUT OF THE CONDUCTOR.}

Figure 1 shows the general lay-out of the conductor. It consists of $N$ concentric regions, with region $i$ from $\mathbf{r}_{\mathrm{i}-1}$ to $\mathbf{r}_{\mathbf{i}}$. Each region, contains a different (composite) material characterized by the constitutive equation, i.e. by the values of $\sigma_{1}, \sigma_{\|}$and $\eta_{s}$. An example of such a conductor is given in figure 2 . Depending on $\sigma_{\downarrow}, \sigma_{\|}$and $\eta_{s}$ we distinguish three types: I-regions, A-regions or S-regions.

\section{I-regions: normal material with isotropic conductivity.}

These regions contain normal material with isotropic conductivity $\sigma$. After entering $\eta_{\mathrm{s}}=0, \sigma_{\perp}=\sigma$ and $\sigma_{\|}=\sigma$ in the constitutive equation we get the usual relation $\mathbf{j}=\sigma \mathbf{E}$. Therefore, $\nabla \cdot \mathbf{j}=0$ implies $\nabla \cdot \mathbf{E}=0$, giving

$$
\partial_{\mathbf{r}} \mathbf{r}+\mathscr{E}_{\mathbf{\varphi}}+\mathscr{E}_{\varphi}=0
$$

The solution of $(5,6,7)$ is

$$
\left(\mathscr{E}_{\mathrm{r}}, \mathscr{E}_{\varphi}, \mathscr{E}\right)=\left(a \mathrm{ar}^{-2}-\mathrm{b}, \mathrm{ar}^{-2}+\mathrm{b},-\beta \mathrm{r}\right),
$$

where $\mathrm{a}$ and $\mathrm{b}$ are constants to be determined later from the boundary conditions.

A-regions: normal material, anisotropic conductivity.

These are the regions where the combination of $\mathrm{Cu}$ and $\mathrm{CuNi}$ leads to anisotropic properties. There is no superconductor, i.e. $\eta_{\mathrm{s}}=0$. Assuming a fraction $\eta_{\mathrm{h}}$ of $\mathrm{Cu}$ with high conductivity $\sigma_{h}$ and a fraction $1-\eta_{h}$ of $\mathrm{CuNi}$ barriers with low conductivity $\sigma_{l}$, we get

$$
\begin{aligned}
& \sigma_{\perp} \cong\left[\left(1+\eta_{h}\right) /\left(1-\eta_{h}\right)\right] \sigma_{1} \\
& \sigma_{\|}=\eta_{h} \sigma_{h}+\left(1-\eta_{h}\right) \sigma_{1} \cong \eta_{h} \sigma_{h} .
\end{aligned}
$$

The ratio $\sigma_{\|} / \sigma_{\perp}$ can be in the order of 100 . Now, the conservation of current $\nabla \cdot \mathbf{j}=0$ is given by

$$
\partial_{r} \mathbf{r} \sigma_{r r} \mathscr{E}_{r}+\sigma_{\varphi \varphi} \mathscr{E}_{\varphi}+\sigma_{\varphi z} \mathbb{Z}_{Z}=0 \text {. }
$$

By combining this with Eqs. $(5,6)$ and substituting $\sigma_{\mathrm{rr}}$, $\sigma_{\varphi \varphi}$ and $\sigma_{\varphi z}$, we obtain a second-order equation for $\mathscr{E}_{\varphi}$,

$$
-\partial_{\mathrm{r}} \mathrm{r}{ }_{\mathrm{r}} \mathrm{r} \mathscr{E}_{\varphi}+\left\{1+\frac{(\mathrm{m}-1)(\beta \mathrm{r})^{2}}{1+(\beta \mathrm{r})^{2}}\right\} \mathscr{E}_{\varphi}=\frac{(\mathrm{m}-1)(\beta \mathrm{r})^{2}}{1+(\beta \mathrm{r})^{2}}
$$

with $m=\sigma_{\|} / \sigma_{\perp}$. One particular solution is $\delta \varphi=1$. The homogeneous part of Eq. (12) can be reduced further by a change of variables $\mathrm{z}=-(\beta \mathrm{r})^{2}$ to the "hypergeometric equation" (see [5])

$$
\mathrm{z}(1-\mathrm{z}) \partial_{\mathrm{zz}} \mathscr{E}_{\varphi}+2(1-\mathrm{z}) \partial_{\mathrm{z}} \mathscr{E}_{\varphi}+\frac{\mathrm{m}-1}{4} \mathscr{E}_{\varphi}=0
$$

This has two linearly independent solutions $\mathbf{u}_{1}(\mathrm{z})$ and $\mathrm{u}_{2}(\mathrm{z})$, so $\mathscr{E} \varphi(\mathrm{r})$ is equal to $1+\mathrm{a} \cdot \mathrm{u}_{1}\left(-\beta \mathrm{r}^{2}\right)+\mathrm{b} \cdot \mathrm{u}_{2}\left(-\beta \mathrm{r}^{2}\right)$, where the constants $a$ and $b$ follow from the boundary conditions. However, numerical integration of (12) is considerably simpler than evaluation of the functions $\mathrm{u}_{1}$ and $\mathrm{u}_{2}$.

\section{S-regions: superconducting filaments with $d_{\mathrm{fil}} \cong 0$.}

Regions with superconductor are in fact highly anisotropic. The electrical field $E_{\|}$associated with a current flow in the filaments is very small. According to Eq. (3), $E_{\|}$will vanish for unsaturated filaments if $\mathrm{d}_{\text {fil }}$ is small. This condition that $\mathbf{E}$ is transverse to the filament direction is expressed as

$$
\mathscr{E}_{\mathrm{z}}=-\beta \mathrm{r} \mathscr{E}_{\varphi}
$$

and is used to complete $(5,6)$. The solution becomes

$$
\left(\mathscr{E}_{\mathbf{r}}, \mathscr{E}_{\varphi}, \mathscr{E}_{\mathrm{Z}}\right)=(-1,1,-\beta \mathbf{r}) \text {, }
$$

which is a special case of (8) with $a=0$ and $b=1$. The current density in principle consists of a normal and a superconducting part

$$
\left(\mathrm{j}_{\mathrm{r}}, \mathrm{j}_{\varphi}, \mathrm{j} z\right)=\left(\sigma_{\perp} \mathrm{E}_{\mathrm{r}}, \sigma_{\perp} \mathrm{E}_{\varphi}+\eta_{\mathrm{s}} \mathrm{j}_{\mathrm{s}} \sin \psi, \sigma_{\perp} \mathrm{E}_{\mathrm{z}}+\eta_{\mathrm{s}} \mathrm{j}_{s} \cos \psi\right)
$$

Here, $\sigma_{\perp}$ is $\sigma_{\mathrm{m}}\left(1+\alpha \eta_{\mathrm{s}}\right) /\left(1-\alpha \eta_{\mathrm{s}}\right)$, with $\sigma_{\mathrm{m}}$ the conductivity of the matrix, and $\alpha$ a constant between -1 and 1 depending on the resistance of the superconductor-matrix interface. The value of $\sigma_{\|}$is irrelevant now because there is no component of $\mathbf{E}$ in this direction.

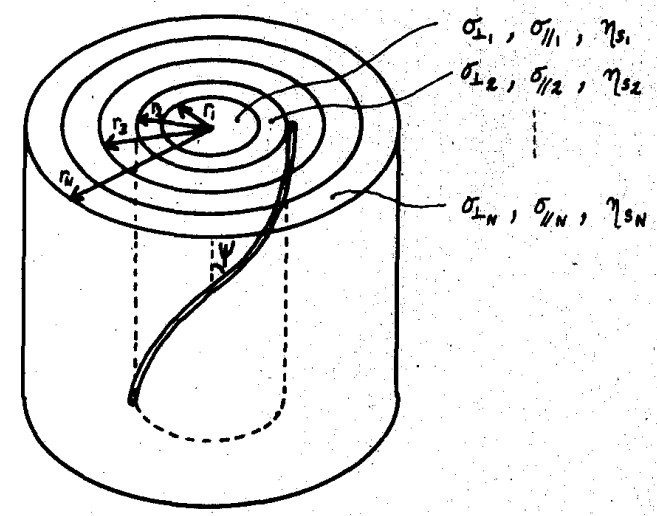

Fig. 1 Geometry of the conductor having concentric regions with different materials. 


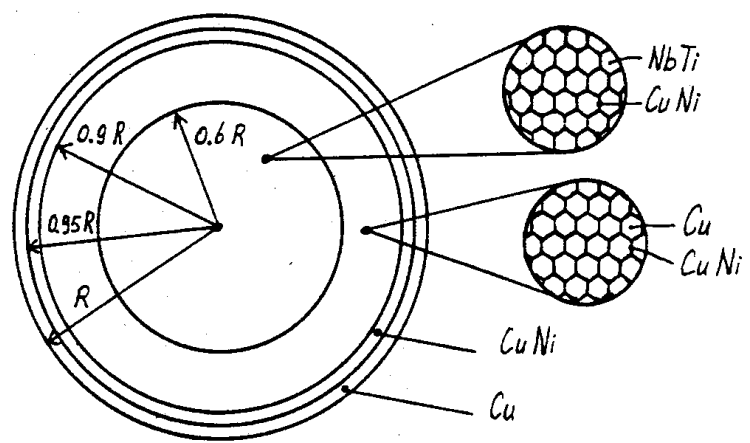

Fig. 2 An example of a conductor with mixed matrix.

\section{BOUNDARY CONDITIONS AND LOSSES}

The boundary conditions to be fulfilled at the interfaces between two regions are continuity of $\mathscr{E}$ and $\sigma_{\perp} \mathscr{E}_{\mathrm{r}}$. In addition we have $\partial_{\mathrm{r}} \mathscr{E}_{\mathrm{r}}=0$ on the axis and $\varphi_{\mathcal{G}=0}$ at the wire surface. This adds up to $2 \mathrm{~N}$ boundary conditions. Note that there are exactly $2 \mathrm{~N}$ unknowns if the conductor consists entirely of I-regions and A-regions. In S-regions, however, there are no unknowns since the solution for $\mathscr{E}$ is given directly by (15), so apparently some of our boundary conditions are superfluous. First, the condition $\partial_{\mathrm{r}} \mathscr{E}_{\mathrm{r}}=0$ at $\mathrm{r}=0$ is automatically satisfied by (15). Secondly, $\sigma_{\perp} \mathscr{E}_{\mathrm{r}}$ need not be continuous at the surface of an S-region, because the current associated with a discontinuity of $\sigma_{\perp} \mathscr{E}_{\mathrm{r}}$ can be taken away in filament direction by a thin layer of current. A closer look shows that actually $\sigma_{\perp} \mathscr{E}_{\mathrm{r}}$ changes continuously within this current-carrying layer, but by neglecting the thickness of the layer we have introduced a jump in $\sigma_{\perp} \mathscr{E}_{\mathrm{r}}$. Note that the thickness of the layer is negligible provided $\overrightarrow{\mathrm{B}}_{\mathrm{a}} \tau<<\mu_{\mathrm{o}} \eta_{\mathrm{s}} \mathrm{j}_{\mathrm{c}} \mathrm{R}$. As an example, consider a conductor with 3 I-regions. The scaled electrical field is

$$
\left(\mathscr{E}_{\mathrm{r}}, \mathscr{E}_{\varphi}, \mathscr{E}_{\mathrm{z}}\right)=\left(\mathrm{a}_{\mathrm{i}} \mathrm{r}^{-2}-\mathrm{b}_{\mathrm{i}}, \mathrm{a}_{\mathrm{i}} \mathrm{r}^{-2}+\mathrm{b}_{\mathrm{i}},-\beta \mathrm{r}\right),
$$

where the index $\mathrm{i}$ refers to region $\mathrm{i}$. The 6 boundary conditions in this case read:

$$
\begin{aligned}
& \left.\partial_{\mathbf{r}} \mathscr{E}_{r=0}\right|_{r=0}=a_{1}=0 \\
& \sigma_{\perp} \mathscr{E}_{\mathrm{r}} \text { at } \mathrm{r}_{1} \Rightarrow \sigma_{1}\left(\mathrm{a}_{1}-\mathrm{b}_{1} \mathbf{r}_{1}^{2}\right)=\sigma_{2}\left(\mathrm{a}_{2}-\mathrm{b}_{2} \mathrm{r}_{1}^{2}\right) \\
& \mathscr{E}_{\varphi} \text { at } \mathrm{r}_{1} \Rightarrow a_{1}+b_{1} \mathrm{r}_{1}^{2}=a_{2}+b_{2} r_{1}^{2} \\
& \sigma_{\perp} \mathscr{E}_{r} \text { at } r_{2} \Rightarrow \sigma_{2}\left(a_{2}-b_{2} r_{2}^{2}\right)=\sigma_{3}\left(a_{3}-b_{3} r_{2}{ }^{2}\right) \\
& \mathscr{E}_{\varphi} \text { at } \mathrm{r}_{2} \quad \Rightarrow \quad a_{2}+b_{2} r_{2}^{2}=a_{3}+b_{3} r_{2}^{2} \\
& \sigma_{\perp} \mathscr{E}_{r} \text { at } r_{3} \Rightarrow a_{3}-b_{3} r_{3}^{2}=0
\end{aligned}
$$

Now, if region 2 were superconductive, we simply drop Eqs. (19) and (21) and replace them by $a_{2}=0$ and $b_{2}=1$. This method of getting the appropriate boundary conditions can be expanded to any number of S-regions. After solving the unknowns $a_{i}$ and $b_{j}$, the coupling loss can be computed. The local loss per unit volume is $\mathbf{j} \cdot \mathbf{E}$, so

$$
\mathrm{p}=\sigma_{\perp}\left(\mathrm{E}_{\mathbf{r}}^{2}+\mathrm{E}_{\perp}^{2}+\mathrm{E}_{\|}^{2}\right)+\left(\sigma_{\|}-\sigma_{\perp}\right) \mathrm{E}_{\|}^{2}+\eta_{\mathrm{s}} \mathrm{j}_{\mathrm{s}} \mathrm{E}_{\|},
$$

where the last two terms vanish, in S-regions because $\mathrm{E}_{\|}=0$, and in I-regions because $\sigma_{\|}=\sigma_{\perp}$ and $\eta_{\mathrm{s}}=0$. Thus, the dissipation per unit length in region $i$ becomes

$$
\begin{aligned}
P_{i} & =\int_{r_{i-1}}^{r_{i}} \int_{0}^{2 \pi} \sigma_{i}{ }_{\perp}\left(E_{r}^{2}+E_{\varphi}^{2}+E_{z}^{2}\right) r d \varphi d r=\sigma_{i_{\perp}}\left(\dot{B}_{a} / \beta\right)^{2} . \\
& \pi\left(r_{i}^{2}-r_{i-1}^{2}\right) \cdot\left(\frac{a_{i}^{2}}{r_{i}^{2} \cdot r_{i-1}^{2}}+b_{i}^{2}+\frac{\beta^{2}\left(r_{i}^{2}+r_{i-1}^{2}\right)}{4}\right)
\end{aligned}
$$

If A-regions are also present, numerical integration of (12) and (18) is required. In that case, the boundary conditions are chosen in the same way, by discarding the two equations associated with continuity of $\sigma_{\perp} \mathscr{E}_{\mathrm{r}}$ for each superconducting region.

\section{RESULTS}

First consider a single A-region. Figure 3 shows the loss in this case after scaling by $\mathrm{P}_{\mathrm{sc}}$, the loss occurring in an S-region with the same $\sigma_{\perp}$ :

$$
P_{s c}=\sigma_{\perp}\left(\dot{B}_{a} / \beta\right)^{2} \cdot \pi R^{2} \cdot\left(1+(\beta R / 2)^{2}\right)
$$

It is clear from the figure that the difference between $A-$ and S-regions disappears for large $\beta R$ and $m$. This is understandable because a region with superconducting filaments is very anisotropic, and on the other hand, normal regions with high anisotropy behave almost as superconductors. The electrical field is plotted in Fig. 4. An interesting aspect is the decrease of $\mathscr{E}_{\|}$ with growing $\mathrm{m}$, implying that $\mathbf{E}$ is almost perpendicular to the direction of twist, except near the surface of the wire where $\mathscr{E}_{\|}$is relatively large and $\mathscr{E}_{\mathrm{r}}$ becomes zero. This is comparable with the thin saturated layer of current at the surface of a superconducting region.

Another example is the conductor shown in Fig. 2, with an A-region enclosed between S- and I-regions. The calculated electrical field in this case is given in Fig. 5. It should be noticed that the jump of $\&$ at the boundary of 1 and 2 decreases with growing anisotropy of region 2. The coupling loss in the four regions is of the same order of magnitude for this wire.

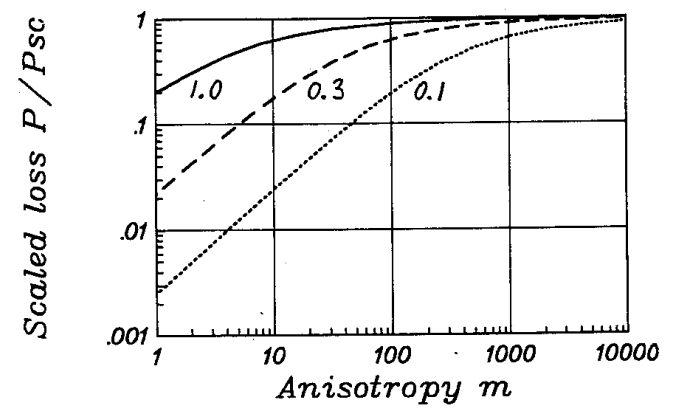

Fig. 3 Scaled coupling loss in a single A-region as a function of the anisotropy $\sigma_{\|} / \sigma_{\perp}$. The curves correspond to $\beta R=0.1,0.3$ and 1.0 . 

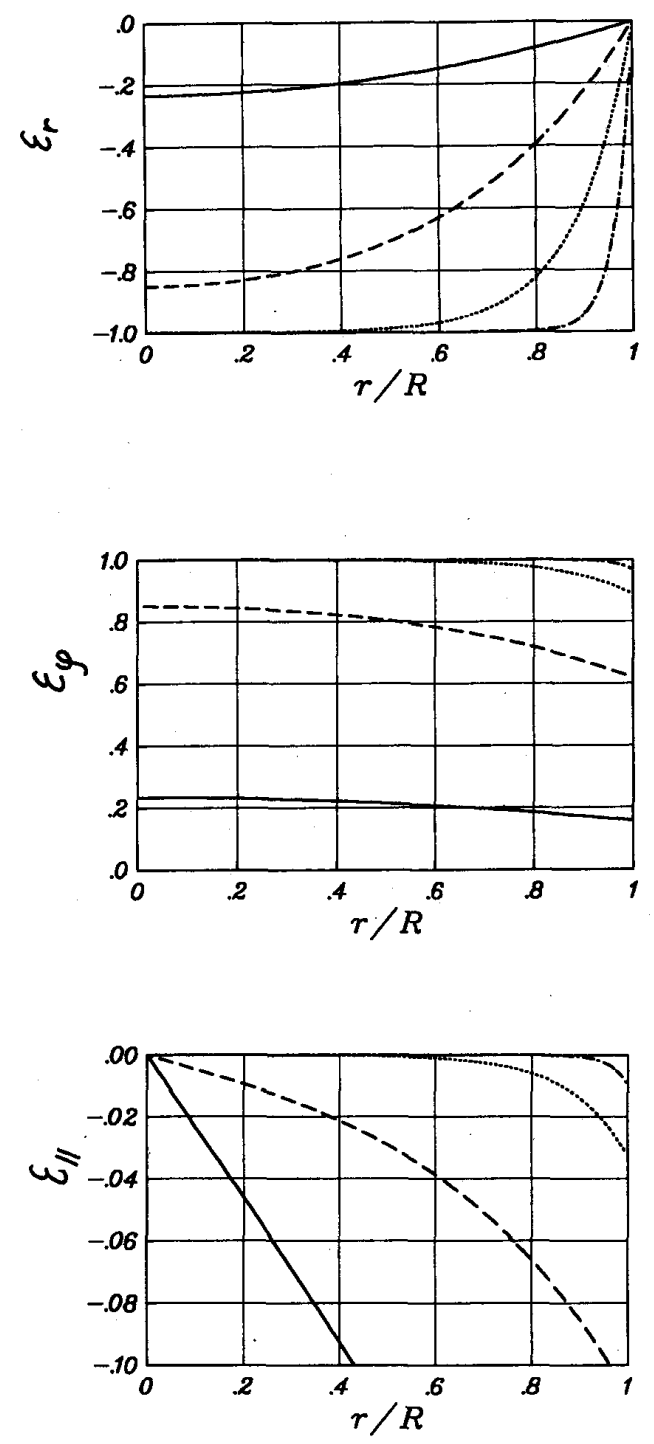

Fig. 4 Scaled electrical field $\mathscr{E}$ in an A-region, with $\beta R=0.3$ and different values of $m$.

- $\mathrm{m}=10 ;-\cdots \mathrm{m}=100 ; \cdots \mathrm{m}=1000 ; \cdots \mathrm{m}=10000$.

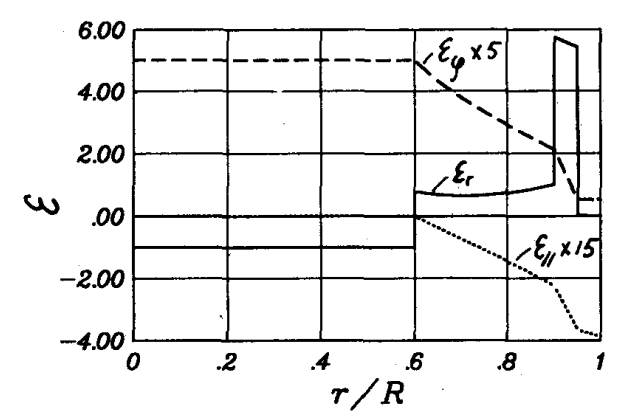

Fig. $5 \mathscr{E}(r)$ for the wire of figure 2 , with $\sigma_{C u}=3 \cdot 10^{9}$, $\sigma_{\mathrm{CuNi}}=3 \cdot 10^{6} \Omega^{-1} \mathrm{~m}^{-1}, \alpha=1$ and $\beta R=0.3$.

\section{CONCLUDING REMARKS}

In the S-regions we imposed $\mathrm{E}_{\|}=0$ in order to solve $\mathbf{E}$, and using $\nabla \cdot \mathbf{j}=0$ we concluded that a thin layer of current-carrying filaments is present at the interfaces with other regions. In the A-regions, on the other hand, we needed the equation $\nabla \cdot \mathbf{j}=0$ in order to solve $\mathbf{E}$. The solution obtained in this way becomes more and more similar to that of the S-region as the anisotropy $\sigma_{\|} / \sigma_{\perp}$ increases, i.e. $E_{\|}$is approximately zero everywhere in the wire except in a thin layer at the surface.

An improvement of the model is to take into account the thickness of the superconducting filaments. Eq. (3) shows that the conductivity along the filaments is finite due to their dynamic resistance when subjected to a changing field. Therefore, the S-regions can also be treated as A-regions using an effective $\sigma_{\|}$:

$$
\sigma_{\|} \cong\left(1-\eta_{s}\right) \sigma_{m}+\eta_{s} j_{s} /\left(\frac{4}{3 \pi} d_{f i l}\left|\dot{B}_{a}\right|\right)
$$

With the described method, the coupling loss can be calculated for a conductor of very general geometry. In particular the effect of anisotropic normal material was taken into account so that mixed matrix conductors can be dealt with, enabling for example an optimization of the amounts and location of the $\mathrm{NbTi}, \mathrm{Cu}$ and $\mathrm{CuNi}$. $\mathrm{A}$ disadvantage is that we limited ourselves to the lowfrequency limit, although for most purposes this is sufficiently accurate.

\section{REFERENCES}

[1] W.J. Carr, Jr., "AC loss in a twisted filamentary superconducting wire," J. Appl. Phys., vol. 45, 1974, pp. 929-934.

[2] P.C. Rem, et al., "A simulation of a multifilamentary wire carrying a transport current in an AC applied field," in Proc. ICEC-11, Butterworth Ltd, Guildford, 1986, pp. 751-755.

[3] A. Février, "Losses in a twisted multifilamentary superconducting composite submitted to any space and time variation of the electromagnetic surrounding," Cryogenics, vol. 23, 1983, pp. 185-200.

[4] L.J.M. van de Klundert, E.M.J. Niessen and P.J. Zandbergen, Electromagnetic response of composite superconducting wires, J. of Eng. Math., vol. 26, 1992, pp. 231-265.

[5] I.S. Gradsteyn and I.M.Ryzhik, Table of integrals, series and products, editor Alan Jeffrey, Academic Press Inc., London, 1980. 